\title{
Bayesian Value-at-Risk with Product Partition Models ${ }^{1}$
}

\author{
Giacomo Bormetti a,b, Maria Elena De Giuli ${ }^{\text {c, }}$ \\ Danilo Delpini $^{\mathrm{d}, \mathrm{b}}$, Claudia Tarantola ${ }^{\mathrm{c}}$ \\ ${ }^{a}$ Institute for Advanced Studies - Center for Risk and Security Study \\ Viale Lungo Ticino Sforza 56, 27100 Pavia, Italy \\ ${ }^{\mathrm{b}}$ National Institute of Nuclear Physics - Pavia Unit \\ Via A.Bassi 6, 27100 Pavia, Italy \\ ${ }^{\mathrm{c}}$ Department of Economics and Quantitative Methods, University of Pavia \\ Via San Felice 5, 27100 Pavia, Italy \\ ${ }^{\mathrm{d}}$ Department of Nuclear and Theoretical Physics, University of Pavia \\ Via A.Bassi 6, 27100 Pavia, Italy
}

\begin{abstract}
In this paper we propose a novel Bayesian methodology for Value-at-Risk computation based on parametric Product Partition Models. Value-at-Risk is a standard tool to measure and control the market risk of an asset or a portfolio, and it is also required for regulatory purposes. Its popularity is partly due to the fact that it is an easily understood measure of risk. The use of Product Partition Models allows us to remain in a Normal setting even in presence of outlying points, and to obtain a closed-form expression for Value-at-Risk computation. We present and compare two different scenarios: a product partition structure on the vector of means and a product partition structure on the vector of variances. We apply our methodology to an Italian stock market data set from Mib30. The numerical results clearly show that Product Partition Models can be successfully exploited in order to quantify market risk exposure. The obtained Value-at-Risk estimates are in full agreement with Maximum Likelihood approaches, but our methodology provides richer information about the clustering structure of the data and the presence of outlying points.
\end{abstract}

Key words: Markov Chain Monte Carlo, Monte Carlo Simulation, Outliers Detection, Parametric Product Partition Models, Value-at-Risk

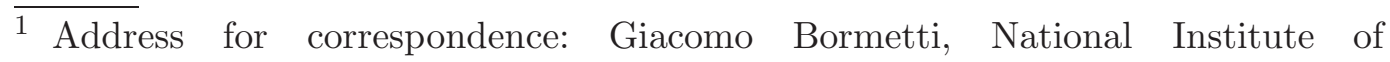
Nuclear Physics - Pavia Unit, Via A. Bassi 6, 27100 Pavia, Italy, Giacomo.Bormetti@pv.infn.it, tel: 0382 987703, fax: 0382526938. 


\section{Introduction}

Following the increase in financial uncertainty, there has been intensive research from financial institutions, regulators and academics to develop models for market risk evaluation. A common and easily understood measure of risk is Value-at-Risk (VaR). In particular, Basel accords impose that all financial institutions have to meet capital requirements based on VaR estimates, see Basel Committee (2006).

$\mathrm{VaR}$ is defined as the maximum potential loss of an asset or a portfolio, at a given time horizon and significance level. An accurate estimate of VaR is important for both banks and regulators. An underestimation of risk could obviously cause problems for banks and other participants in financial markets (e.g. bankruptcy). On the other hand, an overestimation of risk may cause one to allocate too much capital as a cushion for risk exposures, having a negative effect on profits. The Committee does not prescribe banks a special type of model, leaving them free to specify their own model for VaR estimation. In the literature a wide range of models to measure $\mathrm{VaR}$ and to determine the level of regulatory capital are described. For a review on VaR models see e.g. Jorion (2001), Manganelli and Engle (2004) and the remaining list of VaR contributions at the web site http://www.gloriamundi.org.

In this paper we propose a novel Bayesian methodology for VaR estimation based on parametric Product Partition Models (PPMs), and we compare our results with those obtained with standard approaches based on Maximum Likelihood (ML) techniques, see e.g. Mina and Xiao (2001), Mattedi et al. (2004), Bormetti et al. (2007) . In our analysis we pay particular attention in evaluating the statistical uncertainty associated with different results; in fact a good risk management requires not only a pointwise VaR estimate but also an assessment of how much precise the estimate is.

If the returns are independent and identically normally distributed a closedform and easy to implement expression for VaR can be used. Unfortunately, these assumptions fail to be effective for low liquidity markets and short time horizons and have to be relaxed. Possible solutions are to resort to heavy tailed distributions or to abandon the hypothesis of identically distributed returns. In this paper we follow the latter approach and we use a Bayesian methodology based on parametric PPMs. We assume that the returns follow a Normal distribution with a partition structure on the parameters of interest. We assign a prior distribution on the space of all possible partitions and we identify clusters of returns sharing the same mean and variance values. Returns belonging to different clusters are characterised by different values either of the mean or the variance. The hypothesis of identical distribution holds within but non between clusters. As a consequence we abandon the assumption of iden- 
tical distribution while preserving the Normal setting. Furthermore, the use of a product partition approach allows us not only to accommodate anomalous observations but also provides as a by-product a useful tool for their identification; see Quintana and Iglesias (2003), Quintana et al. (2005) and De Giuli et al. (2009) for further details.

We propose and compare two different PPMs for VaR estimation. In the first one we impose a partition structure on the vector of means whereas the volatility is a common random variable; in the second one we impose a partition structure on the vector of variances with common unknown mean. The first approach is quite effective for VaR estimation, but it is very sensitive to the values of prior parameters and even a hierarchical model can not reduce this sensitivity. This problem can be overcame by fixing the values of the hyperparameters according to analysts' experience about the market behaviour. This drawback effect is strongly reduced by imposing a partition structure on the vector of variances. Our results are compared with those obtained with the parametric PPM developed in Loschi et al. (2003) for the identification of change-points in financial time series.

To obtain the posterior distribution of the quantity of interest we use Markov Chain Monte Carlo (MCMC) techniques. MCMC methods have a history in mathematical physics dating back to the algorithm of Metropolis et al. (1953), later generalised by Hastings (1970). In our work we extensively resort to a specific type of Markov chain algorithm, introduced by Geman and Geman (1984) and Gelfand and Smith (1990) and known as Gibbs sampler. The Gibbs sampling algorithms considered here are described in details in sections 3.1 and 3.2. For a recent Bayesian application of Gibbs sampling in the context of financial analysis, see e.g. Chang and Feigenbaum (2008).

The paper is organized as follows. In section 2 we briefly introduce VaR as a measure of risk and parametric PPMs. In section 3 we present two models for VaR estimate and introduce a closed-form expression for VaR computation extending the usual Gaussian form. In section 4 we describe how to exploit the clustering structure induced by PPMs in order to identify outlying points. In section 5 we apply our methodologies to a Mib30 data set and provide a sensitivity analysis of our results with respect to different choices of hyperparameters. Section 6 closes the paper with some final remarks. 


\section{Background and Preliminaries}

\subsection{Value-at-Risk}

$\mathrm{VaR}$ is referred to the probability of extreme losses due to adverse market movements. In particular, for a given significance level $\alpha$ (typically $1 \%$ or $5 \%$ ), VaR is defined as the maximum potential loss over a fixed time horizon of individual assets and portfolios of assets as well. In the following we focus on VaR for a single asset.

If the returns are independent and identically normally distributed with mean $\mu$ and variance $\sigma^{2}$, a closed-form expression for VaR normalised to the spot price is given by

$$
\frac{\Lambda}{W_{0}}=-\mu+\sigma \sqrt{2} \operatorname{erfc}^{-1}(2 \alpha)
$$

where $\Lambda$ is $\mathrm{VaR}, W_{0}$ is the spot price and $\operatorname{erfc}^{-1}$ is the inverse of the complementary error function. In the following, with $\mathrm{VaR}$ we shall refer to the quantity $\Lambda / W_{0}$, if not specified otherwise. If this quantity is expressed in percentage term we name it percentage $\operatorname{VaR}, \operatorname{VaR}(\%)$.

In order to estimate the parameters $\mu$ and $\sigma$ in equation (11), we apply a Bayesian approach based on parametric PPMs; the details are provided in the following section.

\subsection{Parametric Product Partition Models}

We now briefly review the theory of parametric PPMs with reference to our specific problem. For a detailed and more general presentation see Hartigan (1990), Barry and Hartigan (1992).

Let $\boldsymbol{y}=\left(y_{1}, \ldots, y_{t}, \ldots y_{T}\right)$ denote the vector of returns of a generic asset at different time points $t$. The returns are independent, and jointly distributed with probability density function $f$ parameterised by the vector $(\boldsymbol{\theta}, \psi)$. The elements of $\boldsymbol{\theta}$ depend on the time point $t, \boldsymbol{\theta}=\left(\theta_{1}, \ldots, \theta_{T}\right)$, whereas $\psi$ is a parameter that is common to all observations. We consider the following model

$$
\boldsymbol{y} \mid(\boldsymbol{\theta}, \psi) \sim f(\boldsymbol{y} \mid \boldsymbol{\theta}, \psi), \quad \text { with } \quad y_{t} \stackrel{i n d}{\sim} f\left(y_{t} \mid \theta_{t}, \psi\right) \quad t=1, \ldots, T
$$


Given the model in (2), let $S_{0}=\{t: t=1, \ldots, T\}$ be the set of all time periods. A partition of the set $S_{0}, \rho=\left\{S_{1}, \ldots, S_{d}, \ldots, S_{|\rho|}\right\}$ with cardinality $|\rho|$, is defined by the property that $S_{d} \cap S_{d^{\prime}}=\emptyset$ for $d \neq d^{\prime}$ and $\cup_{d} S_{d}=S_{0}$. The generic element of $\rho$ is $S_{d}=\left\{t: \theta_{t}=\theta_{d}^{*}\right\}$, where $\boldsymbol{\theta}^{*}=\left(\theta_{1}^{*}, \ldots, \theta_{|\rho|}^{*}\right)$ is the vector of the unique values of $\boldsymbol{\theta}=\left(\theta_{1}, \ldots, \theta_{T}\right)$. All $\theta_{t}$ whose subscripts $t$ belong to the same set $S_{d} \in \rho$ are (stochastically) equal, in this sense they are regarded as a single cluster.

We assign to each partition $\rho$ the following prior distribution

$$
P\left(\rho=\left\{S_{1}, \ldots, S_{|\rho|}\right\}\right)=K \prod_{d=1}^{|\rho|} C\left(S_{d}\right)
$$

where $C\left(S_{d}\right)$ is a cohesion function and $K$ is the normalising constant. Equation (3) is referred to as the product distribution for partitions. The cohesions represent prior weights on group formation and formalise our opinion on how tightly clustered the elements of $S_{d}$ would be.

The cohesions can be specified in different ways. A useful choice is

$$
C\left(S_{d}\right)=c \times\left(\left|S_{d}\right|-1\right) !
$$

where $c$ is a positive constant and $\left|S_{d}\right|$ denotes the cardinality of the set $S_{d}$. For moderate values of $c$, e.g. $c=1$, the cohesions in equation (4) yield a prior distribution that favours the formation of partitions with a reduced number of large subsets. For more details on the choice of $c$ see e.g. Liu (1996), Quintana and Iglesias (2003), Quintana et al. (2005) and Tarantola et al. (2008).

If non contiguous clusters are considered we can exploit an interesting connection between parametric PPMs and the class of Bayesian nonparametric models with a Dirichlet Process prior, see Antoniak (1974). Under the latter prior, the marginal distribution of the observables is a specific PPM with the cohesion functions specified by equation (4), see Quintana and Iglesias (2003). In this case we can use efficient Markov Chain Monte Carlo (MCMC) algorithms developed for Bayesian nonparametric problems.

When dealing with contiguous blocks, as in the change-point problem, this connection cannot be exploited, and specific MCMC algorithms are required, see e.g. Loschi et al. (2003). 


\section{$3 \quad$ VaR Computation via Product Partition Models}

Let $\boldsymbol{y}$ be the vector of daily returns of a generic asset. We assume that the returns are normally distributed with parameter vector $(\boldsymbol{\theta}, \psi)$. We present and compare two different PPMs; in the first one we impose a partition structure on the vector of means, and in the second one we consider partitions on the vector of variances. In the following the PPM applied to the vector of means will be shortly referred to as the $\boldsymbol{\mu}$-PPM approach, while $\boldsymbol{\sigma}^{2}$-PPM will refer to the PPM for the vector of variances. In $\boldsymbol{\mu}$-PPM the vector $\boldsymbol{\theta}$ is the vector of means while in $\boldsymbol{\sigma}^{2}$-PPM it corresponds to the vector of variances. In the former model $\psi$ is the variance and in the latter it corresponds to the mean.

We consider the following hierarchical structure

$$
\begin{aligned}
& y_{t} \mid\left(\rho,\left(\theta_{1}^{*}, \ldots, \theta_{|\rho|}^{*}\right), \sigma^{2}\right) \stackrel{i n d .}{\sim} N\left(y_{t} \mid\left(\theta_{t}, \psi\right)\right) \\
& \theta_{1}^{*}, \ldots, \theta_{|\rho|}^{*} \mid(\rho, \psi) \stackrel{i . i . d .}{\sim} f(\cdot \mid \psi), \\
& \rho \sim \operatorname{product} \text { distribution, with } C\left(S_{d}\right)=c \times\left(\left|S_{d}\right|-1\right) ! \\
& \psi \sim g(\psi),
\end{aligned}
$$

where $f$ and $g$ denote generic density functions and the product distribution is defined in equation (3).

The elicitation of a partition structure on the vector of means (or variances) allows us to remain in a Normal setting without assuming identical distribution of the returns. An alternative model that could be used to take into account atypical returns when estimating VaR consists of assuming $t$-distributed rather than Normal data. This analysis has been performed by Quintana and Iglesias (2003) in the context of regression models, showing that parametric PPMs in a Normal setting are even more effective if the purpose is to deal and identify extreme values.

In sections 3.1 and 3.2 we describe in details our models and in section 3.3 we propose a closed-form expression for VaR computation.

\subsection{Product Partition Models on Vector of Means}

In the $\boldsymbol{\mu}$-PPM approach we impose a partition structure on the vector of means $\boldsymbol{\mu}=\left(\mu_{1}, \ldots, \mu_{T}\right)$. By inducing a cluster structure on the vector $\boldsymbol{\mu}$ we try to accommodate for atypical $y_{t}$ values. In order to achieve this goal we use the following hierarchical model 


$$
\begin{aligned}
& y_{t} \mid\left(\rho,\left(\mu_{1}^{*}, \ldots, \mu_{|\rho|}^{*}\right), \sigma^{2}\right) \stackrel{\text { ind. }}{\sim} N\left(\mu_{t}, \sigma^{2}\right) \\
& \mu_{1}^{*}, \ldots, \mu_{|\rho|}^{*} \mid\left(\rho, \sigma^{2}\right) \stackrel{\text { i.i.d. }}{\sim} N\left(m, \tau_{0}^{2} \sigma^{2}\right), \\
& \rho \sim \text { product distribution, with } C\left(S_{d}\right)=c \times\left(\left|S_{d}\right|-1\right) ! \\
& \sigma^{2} \sim I G\left(\nu_{0}, \lambda_{0}\right),
\end{aligned}
$$

where $\boldsymbol{\mu}^{*}=\left(\mu_{1}^{*}, \ldots, \mu_{|\rho|}^{*}\right)$ is the vector of all entries of $\boldsymbol{\mu}$ for a given partition $\rho$, and $I G\left(\nu_{0}, \lambda_{0}\right)$ is an Inverted Gamma distribution with $\mathbb{E}\left[\sigma^{2}\right]=\lambda_{0} /\left(\nu_{0}-1\right)$, $\nu_{0}>1$ and $\lambda_{0}>0$.

The complete joint distribution for the model is given by

$$
\begin{aligned}
& f\left(\boldsymbol{y}, \boldsymbol{\mu}, \rho=\left\{S_{1}, \ldots, S_{|\rho|}\right\}, \sigma^{2}\right) \propto \exp \left\{-\frac{1}{2 \sigma^{2}} \sum_{d=1}^{|\rho|} \sum_{t \in S_{d}}\left(y_{t}-\mu_{d}^{*}\right)^{2}\right\} \\
& \quad \times \exp \left\{-\frac{1}{2 \tau_{0}^{2} \sigma^{2}} \sum_{d=1}^{|\rho|}\left(\mu_{d}^{*}-m\right)^{2}-\frac{\lambda_{0}}{\sigma^{2}}\right\} \frac{1}{\left(\sigma^{2}\right)^{1+\nu_{0}+\frac{T+\rho}{2}}} \prod_{d=1}^{|\rho|}\left(\left|S_{d}\right|-1\right) ! .
\end{aligned}
$$

To fit this model we adapt an algorithm proposed by Bush and MacEachern (1996) in the context of Bayesian nonparametric inference. Once a starting value for the vector $\boldsymbol{\mu}$ has been provided, we iteratively sample from the joint posterior distribution of model and parameters by means of the Gibbs algorithm described below.

Step (i) : Sample $\sigma^{2}$ from its full conditional distribution

$$
\sigma^{2} \mid \boldsymbol{\mu}, \boldsymbol{y} \sim I G\left\{\nu_{0}+\frac{T}{2}+\frac{|\rho|}{2}, \lambda_{0}+\frac{1}{2 \tau_{0}^{2}} \sum_{d=1}^{|\rho|}\left(\mu_{d}-m\right)^{2}+\frac{1}{2} \sum_{t=1}^{T}\left(y_{t}-\mu_{t}\right)^{2}\right\} .
$$

Step (ii) : Update each $\mu_{t}, t=1, \ldots, T$, by sampling from the mixture

$$
\mu_{t} \mid \boldsymbol{\mu}_{-t}, \sigma^{2}, \boldsymbol{y} \sim \sum_{j \neq t} q_{t j} \delta_{\mu_{j}}\left(\mu_{t}\right)+q_{t 0} \times N\left(\frac{y_{t} \tau_{0}^{2}+m}{1+\tau_{0}^{2}}, \frac{\sigma^{2} \tau_{0}^{2}}{1+\tau_{0}^{2}}\right),
$$

where $\boldsymbol{\mu}_{-t}$ is obtained from $\boldsymbol{\mu}$ by removing the $t$-th entry and $\delta_{\mu_{j}}\left(\mu_{t}\right)$ is the Dirac delta centered on $\mu_{t}$.

The distribution in equation (6) corresponds to a mixture of point masses and a Normal distribution, with weights

$$
\begin{aligned}
& q_{t j} \propto \exp \left\{-\frac{1}{2 \sigma^{2}}\left(y_{t}-\mu_{j}\right)^{2}\right\} \\
& q_{t 0} \propto \frac{c}{\sqrt{1+\tau_{0}^{2}}} \exp \left\{-\left(y_{t}-m\right)^{2} /\left[2 \sigma^{2}\left(1+\tau_{0}^{2}\right)\right]\right\} \\
& \sum_{j \neq t} q_{t j}+q_{t 0}=1 .
\end{aligned}
$$


Step (iii) : Before proceeding to the next Gibbs iteration we update the vector $\boldsymbol{\mu}^{*}$, given the partition $\rho$, sampling from

$$
\mu_{d}^{*} \sim N\left(\frac{\sum_{t \in S_{d}} y_{t}+m / \tau_{0}^{2}}{\left|S_{d}\right|+1 / \tau_{0}^{2}}, \frac{\sigma^{2}}{\left|S_{d}\right|+1 / \tau_{0}^{2}}\right) \quad d=1, \ldots,|\rho| .
$$

This last step was introduced in Bush and MacEachern (1996) to avoid being trapped in sticky patches in the Markov space.

The weights $q_{t j}$ represent the finite probability of replacing $\mu_{t}$ with a value $\mu_{j}$ already belonging to the vector of means. On the other hand $q_{t 0}$ represents the finite probability of replacing the old $\mu_{t}$ value with a newly sampled one. It is worth noticing again the role played by the constant $c$. A greater value of $c$ increases the probability to generate new values. Generally, the higher $c$ is, the higher the probability to obtain an elevate number of clusters will be.

As it turns out from the empirical analysis, see section 5, posterior distributions are quite sensitive to the value of the parameter $\lambda_{0}$ of the Inverted Gamma distribution in the hierarchical model defined in (5). We tried to reduce this drawback effect by introducing a hyperprior distribution on $\lambda_{0}$. This translates into a minor modification of model (5)

$$
\begin{aligned}
& y_{t} \mid\left(\rho,\left(\mu_{1}^{*}, \ldots, \mu_{|\rho|}^{*}\right), \sigma^{2}, \lambda_{0}\right) \stackrel{i n d .}{\sim} N\left(\mu_{t}, \sigma^{2}\right) \\
& \mu_{1}^{*}, \ldots, \mu_{|\rho|}^{*} \mid\left(\rho, \sigma^{2}, \lambda_{0}\right) \stackrel{i . i . d .}{\sim} N\left(m, \tau_{0}^{2} \sigma^{2}\right) \\
& \rho \sim \operatorname{product} \text { distribution } \\
& \sigma^{2} \mid \lambda_{0} \sim I G\left(\nu_{0}, \lambda_{0}\right) \\
& \lambda_{0} \sim G(\eta, \phi)
\end{aligned}
$$

where $G(\eta, \phi)$ is a Gamma distribution with $\mathbb{E}\left[\lambda_{0}\right]=\eta \phi, \eta>0$ and $\phi>0$. The previous Gibbs sampling algorithm must be modified coherently. Now we have to provide a starting point for $\sigma^{2}$ too, while Step (i) splits in two sub-steps:

Step (ia) :

$$
\lambda_{0} \mid \boldsymbol{\mu}, \boldsymbol{y}, \sigma^{2} \sim G\left(\nu_{0}+\eta, \frac{\sigma^{2} \phi}{\sigma^{2}+\phi}\right)
$$

Step (ib) :

$$
\sigma^{2} \mid \boldsymbol{\mu}, \boldsymbol{y}, \lambda_{0} \sim I G\left(\nu_{0}+\frac{|\rho|}{2}+\frac{T}{2}, \lambda_{0}+\frac{1}{2 \tau_{0}^{2}} \sum_{d=1}^{|\rho|}\left(\mu_{d}-m\right)^{2}+\frac{1}{2} \sum_{t=1}^{T}\left(y_{t}-\mu_{t}\right)^{2}\right)
$$

Step (ii) and Step (iii) do not change. 


\subsection{Product Partition Models on Vector of Variances}

An alternative way to relax the hypothesis of identical distribution of the returns, without renouncing to the normality assumption, is to promote the variance $\sigma^{2}$ from a scalar to the vectorial quantity $\boldsymbol{\sigma}^{\mathbf{2}}=\left(\sigma_{1}^{2}, \ldots, \sigma_{T}^{2}\right)$ and to impose a clustering structure on $\sigma^{2}$. Our aim is to create clusters of observations, not necessarily contiguous in time, sharing the same value $\sigma_{d}^{2^{*}}$ of the variance.

We consider the following hierarchical model

$$
\begin{aligned}
& y_{t} \mid\left(\mu,\left(\sigma_{1}^{2^{*}} \ldots \sigma_{|\rho|}^{2^{*}}\right), \rho\right) \stackrel{\text { ind. }}{\sim} N\left(\mu, \sigma_{t}^{2}\right), \\
& \mu \mid\left(\left(\sigma_{1}^{2^{*}} \ldots \sigma_{|\rho|}^{2^{*}}\right), \rho\right) \sim N\left(m, \frac{\lambda_{0}}{T\left(\nu_{0}-1\right)}\right), \\
& \sigma_{1}^{2^{*}} \ldots \sigma_{|\rho|}^{2^{*}} \mid \rho \stackrel{i . i . d .}{\sim} I G\left(\nu_{0}, \lambda_{0}\right), \\
& \rho \sim \text { product distribution, with } C\left(S_{d}\right)=c \times\left(\left|S_{d}\right|-1\right) !,
\end{aligned}
$$

with the variance of the Normal prior over $\mu$ equal to $\lambda_{0} /\left[T\left(\nu_{0}-1\right)\right]$, where $\lambda_{0} /\left(\nu_{0}-1\right)$ is the first moment of $I G\left(\nu_{0}, \lambda_{0}\right)$ and $1 / T$ ia a scaling factor.

The joint distribution is given by

$$
\begin{aligned}
& f\left(\boldsymbol{y}, \mu, \rho=\left\{S_{1}, \ldots, S_{|\rho|}\right\}, \boldsymbol{\sigma}^{2}\right) \propto \exp \left\{-\frac{T\left(\nu_{0}-1\right)}{2 \lambda_{0}}(\mu-m)^{2}\right\} \\
\times & \exp \left\{-\frac{1}{2} \sum_{d=1}^{|\rho|} \sum_{t \in S_{d}} \frac{\left(y_{t}-\mu\right)^{2}}{\sigma_{d}^{2 *}}-\sum_{d=1}^{|\rho|} \frac{\lambda_{0}}{\sigma_{d}^{2 *}}\right\} \prod_{d=1}^{|\rho|}\left(\sigma_{d}^{2 *}\right)^{-\left(\nu_{0}+1+\left|S_{d}\right| / 2\right)}\left(\left|S_{d}\right|-1\right) ! .
\end{aligned}
$$

In order to sample from the posterior distribution of the model and parameters we use a Gibbs algorithm that is a generalization of the one used in the section 3.1. The algorithm consists of the three steps below.

Step (i) : Sample $\mu$ from its full conditional distribution

$$
\mu \mid \boldsymbol{\sigma}^{2}, \boldsymbol{y} \sim N\left(\frac{m+\sum_{d=1}^{|\rho|} \frac{\lambda_{0}}{T\left(\nu_{0}-1\right) \sigma_{d}^{2 *}} \sum_{i \in S_{d}} y_{i}}{1+\sum_{d=1}^{|\rho|}\left|S_{d}\right| \frac{\lambda_{0}}{T\left(\nu_{0}-1\right) \sigma_{d}^{2 *}}}, \frac{\frac{\lambda_{0}}{T\left(\nu_{0}-1\right)}}{1+\sum_{d=1}^{|\rho|}\left|S_{d}\right| \frac{\lambda_{0}}{T\left(\nu_{0}-1\right) \sigma_{d}^{2 *}}}\right) .
$$

Step (ii) : Update each $\sigma_{t}^{2}, t=1, \ldots, T$, by sampling from the mixture

$$
\sigma_{t}^{2} \mid \boldsymbol{\sigma}_{-t}^{\mathbf{2}}, \boldsymbol{y} \sim \sum_{j \neq t} \tilde{q}_{t j} \delta_{\sigma_{j}^{2}}\left(\sigma_{t}^{2}\right)+\tilde{q}_{t 0} \times I G\left(\nu_{0}+\frac{1}{2}, \lambda_{0}+\frac{\left(y_{t}-\mu\right)^{2}}{2}\right),
$$


where $\boldsymbol{\sigma}_{-t}^{2}$ is obtained from $\boldsymbol{\sigma}^{\mathbf{2}}$ by removing $t$-th entry and $\delta_{\sigma_{j}^{2}}\left(\sigma_{t}^{2}\right)$ is the Dirac delta centered on $\sigma_{t}^{2}$.

The distribution in equation (10) corresponds to a mixture of point masses and an Inverted Gamma distribution, with weights

$$
\begin{aligned}
& \tilde{q}_{t j} \propto \frac{1}{\sqrt{\sigma_{j}^{2}}} \mathrm{e}^{-\frac{\left(y_{t}-\mu\right)^{2}}{2 \sigma_{j}^{2}}}, \\
& \tilde{q}_{t 0} \propto c \times \frac{\Gamma\left(\nu_{0}+\frac{1}{2}\right)}{\Gamma\left(\nu_{0}\right)} \frac{2^{\nu_{0}+\frac{1}{2}}\left(\lambda_{0}\right)^{\nu_{0}}}{\left[\left(y_{t}-\mu\right)^{2}+2 \lambda_{0}\right]^{\nu_{0}+\frac{1}{2}}}, \\
& \sum_{j \neq t} q_{t j}+q_{t 0}=1,
\end{aligned}
$$

where $\Gamma$ is the Euler Gamma function.

Step (iii) : In order to avoid being trapped in sticky regions of the Markov space, resample $\sigma_{d}^{2 *}$ from

$$
\sigma_{d}^{2 *} \sim I G\left(\nu_{0}+\frac{\left|S_{d}\right|}{2}, \lambda_{0}+\sum_{t \in S_{d}} \frac{\left(y_{t}-\mu\right)^{2}}{2}\right) \quad d=1, \ldots,|\rho| .
$$

A well-known stylized fact about volatilities is the bursting effect and PPMs can be exploited to identify change points in volatility time series. This problem has been extensively considered by Loschi et al. (2003), Loschi et al. (2007) and Loschi et al. (2008). Although we do not focus on this aspect here, in the empirical analysis in section 5 we shall use the results from the algorithm by Loschi et al. (2003), labelled $\boldsymbol{\sigma}^{2}$-CP, as a yardstick to be compared with our numerical results.

\subsection{VaR Estimation}

We now present how the posterior distribution of $\mathrm{VaR}$ and consequently its Bayesian estimate can be obtained by using the output of the MCMC algorithms described in sections 3.1 and 3.2 ,

First we focus our attention on the PPM on the vector of means. Let indicate with $\boldsymbol{\mu}_{(\ell)}^{*}=\left(\mu_{1(\ell)}^{*}, \ldots, \mu_{|\rho|}^{*}(\ell)\right)$ and $\sigma_{(\ell)}^{2}$ respectively the vector of means and the variance sampled at the $\ell$-th iteration of the Gibbs algorithm. At each iteration we obtain a peculiar clustering structure. All returns share the same value of $\sigma_{(\ell)}^{2}$, but each cluster is characterized by a different value $\mu_{d(\ell)}^{*}$. In order to provide a single VaR estimate for each iteration of the chain we propose to combine the different entries of $\boldsymbol{\mu}_{(\ell)}^{*}$ by means of an arithmetic average and we consider the following equation 


$$
\frac{\Lambda_{(\ell)}}{W_{0}}=-\sum_{d=1}^{|\rho|} \frac{\left|S_{d(\ell)}\right|}{T} \mu_{d(\ell)}^{*}+\sigma_{(\ell)} \sqrt{2} \operatorname{erfc}^{-1}(2 \alpha) .
$$

It is worth noticing that for trivial partitions, i.e. $|\rho|=1$, equation (11) reduces to the usual expression given in equation (11).

If we impose a clustering structure over the vector of variances, VaR can be computed in an analogous way but the arithmetic average is performed over different values of $\sigma_{d(\ell)}^{*}$, that is

$$
\frac{\Lambda_{(\ell)}}{W_{0}}=-\mu_{(\ell)}+\sum_{d=1}^{|\rho|} \frac{\left|S_{d(\ell)}\right|}{T} \sigma_{d(\ell)}^{*} \sqrt{2} \operatorname{erfc}^{-1}(2 \alpha) .
$$

In this case all returns share the same value of $\mu_{(\ell)}$ but each cluster is characterised by a different value of $\sigma_{d}^{2 *}(\ell)$.

The resulting VaR estimate is obtained as the ergodic mean of the quantities $\Lambda_{(\ell)}$ in (11) or (12) for $\boldsymbol{\mu}$-PPM or $\boldsymbol{\sigma}^{2}$-PPM respectively:

$$
\frac{\Lambda}{W_{0}} \doteq \frac{1}{L} \sum_{\ell=1}^{L} \frac{\Lambda_{(\ell)}}{W_{0}}
$$

Finally, VaR under the $\boldsymbol{\sigma}^{\mathbf{2}}$-CP model is computed in a similar way via equation (13).

\section{Product Partition and Outliers Identification}

PPMs can be a useful tool for outliers identification. Following Quintana and Iglesias (2003), we work in a Bayesian decision theoretical framework and we propose an efficient algorithm for outliers identification. We model outliers as a shift in the mean of the data and consequently we fix our attention on $\boldsymbol{\mu}$-PPM. The extent of that shift is indeed the criterion used by this model to induce a new cluster on the vector of returns, as emerge from the expression of the weights $q_{t j}$ and $q_{t 0}$.

Our aim is to select the partition that best separates the main group of standard observations from one or more groups of atypical data. Each partition corresponds to a different model, and the best model is the one minimising a given loss function. Let $\left(\boldsymbol{\mu}, \sigma^{2}\right)$ be the vector of parameters of the model and $\left(\boldsymbol{\mu}_{\rho}, \sigma_{\rho}^{2}\right)$ the corresponding vector that results when fixing $\rho$. We consider the 
following loss function that combines the estimation of the parameters and the partition selection problems

$$
L\left(\rho, \boldsymbol{\mu}_{\rho}, \sigma_{\rho}^{2}, \boldsymbol{\mu}, \sigma^{2}\right)=\frac{k_{1}}{T}\left\|\boldsymbol{\mu}_{\rho}-\boldsymbol{\mu}\right\|^{2}+k_{2}\left(\sigma_{\rho}^{2}-\sigma^{2}\right)^{2}+\left(1-k_{1}-k_{2}\right)|\rho|,
$$

where $\|\cdot\|$ is the Euclidean norm and $k_{1}, k_{2}$ are non-negative cost-complexity parameters with $k_{1}+k_{2} \leq 1$.

Minimising the expected value of (14) is equivalent to choosing the partition that minimises the following score function

$$
S C(\rho)=\frac{k_{1}}{T}\left\|\hat{\boldsymbol{\mu}}_{B}(\boldsymbol{y})-\hat{\boldsymbol{\mu}}_{\rho}(\boldsymbol{y})\right\|^{2}+k_{2}\left[\hat{\sigma}_{B}^{2}(\boldsymbol{y})-\hat{\sigma}_{\rho}^{2}(\boldsymbol{y})\right]^{2}+\left(1-k_{1}-k_{2}\right)|\rho|,
$$

where the subscript " $B$ " means that we consider the Bayesian estimates of the corresponding parameter whereas the subscript " $\rho$ " indicates the estimate conditionally on a given partition $\rho$. Formally, we have that $\hat{\boldsymbol{\mu}}_{B}(\boldsymbol{y})=\mathbb{E}[\boldsymbol{\mu} \mid \boldsymbol{y}]$, $\hat{\boldsymbol{\mu}}_{\rho}(\boldsymbol{y})=\mathbb{E}[\boldsymbol{\mu} \mid \boldsymbol{y}, \rho]$ and analogously for $\hat{\sigma}_{B}^{2}(\boldsymbol{y})$ and $\hat{\sigma}_{\rho}^{2}(\boldsymbol{y})$. The Bayesian estimates are obtained via the Gibbs sampling algorithm described in the section 3.1. The evaluation of $\hat{\boldsymbol{\mu}}_{\rho}(\boldsymbol{y})$ and $\hat{\sigma}_{\rho}^{2}(\boldsymbol{y})$ also requires the use of the Gibbs sampling scheme, but in a structurally simpler version. Indeed the partition $\rho$ is fixed and we can sample from the joint posterior distribution performing iteratively Step (i) and Step (iii), but skipping Step (ii).

An exhaustive search on the space of all possible partitions is infeasible. In fact, for a set with $T$ elements, the number of all possible partitions is equal to $B(T)$, the Bell number of order $T$, recursively defined by $B(T+1)=\sum_{k=0}^{T}\left(\begin{array}{l}T \\ k\end{array}\right) B(k)$, with $B(0)=1$. This quantity is extremely large even for moderate values of $a$, therefore we need to restrict our search to a tractable subset of all partitions.

In order to find the minimum of the score function in equation (15), we perform an exhaustive search over the partitions with cardinality up to three, selected as follow.

i) Let $\boldsymbol{\mu}_{B}=\left(\mu_{1}, \ldots, \mu_{T}\right)$ be the vector of the Bayesian estimates of the returns means, and $\widetilde{\boldsymbol{\mu}}_{B}=\left(\widetilde{\mu}_{1}, \ldots, \widetilde{\mu}_{\widetilde{T}}\right)$ be the vector of the unique entries of $\boldsymbol{\mu}_{B}$ sorted in increasing order, with $\widetilde{\mu}_{1} \doteq \min \left(\mu_{t}\right), \widetilde{\mu}_{\widetilde{T}} \doteq \max \left(\mu_{t}\right)$, and $\widetilde{T} \leq T$.

ii) We perform our search of the optimal partition over the set of the partitions $\rho=\left\{S_{1}, S_{2}, S_{3}\right\}$, where $S_{1}=\left\{t: \mu_{t}<\widetilde{\mu}_{i}\right\}, S_{2}=\left\{t: \widetilde{\mu}_{i} \leq \mu_{t} \leq \widetilde{\mu}_{j}\right\}$, and $S_{3}=\left\{t: \mu_{t}>\widetilde{\mu}_{j}\right\}$ with $i, j=1, \ldots, \widetilde{T}$. We select as optimal partition the one for which the score function achieves the minimum value in (15).

When $i \neq 1$ and $j \neq \widetilde{T}, \rho$ is a genuine cardinality-3 partition. The indexes in $S_{1}$ and $S_{3}$ may be considered as representative of those returns being in the "left tail" and the "right tail" of the empirical distribution of $\boldsymbol{y} . S_{2}$ corresponds to elements occupying the central region of this distribution. When $i=1$ 
and $j=\widetilde{T}$, we are exploring the trivial partition, $S_{1}=S_{3}=\emptyset$. If $i=1$ or $j=\widetilde{T}$, partitions have just two clusters. However, there is an alternative way to generate cardinality-2 partitions. Given every cardinality-3 partition $\rho$, we consider the new partition $\hat{\rho}=\left\{S_{1}, S_{2}\right\}$, with $S_{1} \doteq S_{1} \cup S_{3}$. This step is necessary for our search to be exhaustive also over the space of cardinality-2 partitions.

Once the optimal partition has been found, we identify the outliers with those elements in $\boldsymbol{y}$ whose indexes belong to the sets with lowest cardinality.

\section{Empirical Analysis of Financial Data}

\subsection{The Data}

The methodologies described in the previous sections are now illustrated and tested over the MIB30 index and its three components with the highest excess of kurtosis, where standard approaches based on Normal distributions usually fail. In particular we apply our analysis to the Italian assets Lottomatica (LTO.MI), Mediobanca (MB.MI) and Snam Rete Gas (SRG.MI). We consider time series of daily returns from April 2004 to March 2008. All time series are made of 1000 daily returns. The data are freely downloadable from the site http://it.finance.yahoo.com.

\subsection{Choice of Hyperparameters and Computational Details}

In the examples below we use the following values of the hyperparameters. In models (5), (17) and (9) we set $m=0$, while $\tau_{0}^{2}=10^{3}$ in (15). The choice for $m$ can be motivated by the fact that in VaR estimation for short time horizon, typically from one day until one week, the value of the mean is usually neglected, see e.g. Mina and Xiao (2001). In the Inverted Gamma distribution we set $\lambda_{0}=0.0101$ and $\nu_{0}=2.01$. With these choices we have prior expectation and variance 0.01 for $\sigma^{2}$, reflecting what is known from the past experience about the volatility behaviour for equity assets. The value of $c$ that controls the clustering structure over the vector of parameters is set to 1 , in order to favour the creation of a small number of large clusters. As far as concern the score function parameters of equation (15) we set $k_{1} \sim 0.996$ and $k_{2} \sim$ 0.002 , giving priority to the estimation of $\boldsymbol{\mu}$, and imposing little restriction on the estimation of the other parameters. For the $\boldsymbol{\sigma}^{2}-\mathrm{CP}$ model that we use as yardstick model we set the priors' parameters following the suggestions given in Loschi et al. (2003). In particular we consider the conjugate Normal- 
Inverted-Gamma model, with the probability $p$ that a change occurs at any instant in the sequence equal to 0.1 .

The programs are written in Fortran 77 language, with basic function of linear algebra provided by BLAS and SLATEC libraries. Random number generators, Normal and Gamma sampling are based on the algorithms implemented in the RANDOM library. The interested reader can download them and find

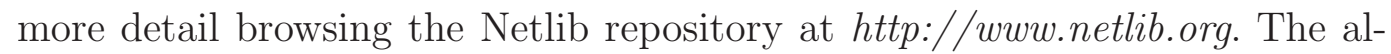
gorithm proposed by Loschi et al. (2003) is freely available at the web site ftp://ftp.est.ufmg.br/pub/loschi/.

We run the MCMC algorithms with 10000 sweeps and a burn-in equal to 1000. Convergence of the MCMC algorithm is assessed using diagnostics implemented in the package BOA, see Smith (2001). All the numerical computations are performed with an AMD Athlon 64 X2 $38002.0 \mathrm{GHz}$ processor and 2.0 GByte of RAM, OS Gentoo Linux kernel 2.6.22. Each program takes nearly 15 minutes to generate the ergodic sample and to compute the parameters posterior distributions. The clustering structure for each step of the chain and the relative frequencies of the partitions are computed by means of sorting algorithms. It takes further 10 minutes to accomplish this task. In our programs we use sorting algorithms implementing strategies of $O\left(T^{2}\right)$ computational complexity. It is possible to reduce the computational burden by means of $O(T \log T)$ algorithms. However it is crucial that sorting preserves relative order of records with equal keys, but this in general requires storage of an auxiliary amount of memory.

\subsection{VaR Results}

In table 1 we report Bayesian estimates of percentage VaR for $\alpha=1 \%$ and $\alpha=5 \%$ and the $68 \%$ posterior credible interval.

\section{TABLE 1 ABOUT HERE}

The estimates of VaR obtained with $\boldsymbol{\sigma}^{2}$-PPM and $\boldsymbol{\sigma}^{2}$-CP are in good agreement even if the two approaches are quite different in spirit. The former approach is a natural extension of the $\boldsymbol{\mu}$-PPM to the vector of variances while the latter one is specific for change point identification.

The PPM on the vector of means in general underestimates VaR with respect to the values given by the PPMs applied to the variances. This fact can be empirically justified noticing that for daily time horizons the contribution to VaR due to the volatility $\sigma$ is of order ten greater than that due to the mean $\mu$. 
Figure 1 depicts posteriors distributions for VaR estimates at level $\alpha=1 \%$. In the first row we present the results based on the $\boldsymbol{\mu}$-PPM approach, while the second corresponds to $\boldsymbol{\sigma}^{2}$-PPM. The posterior distribution of VaR presents a higher variability under the $\boldsymbol{\sigma}^{2}$-PPM approach than under the $\boldsymbol{\mu}$-PPM VaR one.

The posterior expectation of the number of clusters is low for both the $\boldsymbol{\mu}$-PPM and $\boldsymbol{\sigma}^{2}-\mathrm{PPM}$ approaches and, moreover, the partitions are characterised by a very large cluster and few small ones. The results are presented in table 2 .

\section{TABLE 2 ABOUT HERE}

The arithmetic average in equations (11) and (12) is therefore dominated by

the values of $\mu_{d(\ell)}^{*}$ and $\sigma_{d(\ell)}^{2 *}$ that correspond to the largest cluster, while outlying clusters introduce corrections to VaR.

\section{FIGURE 1 ABOUT HERE}

We now compare our results with those obtained with standard parametric approaches based on ML estimators for the mean and variance. In particular we consider results obtained with a Normal model and with the generalised Student-t (GST) distribution, see e.g. Bormetti et al. (2007). In the GST we set the tail index $\nu>2$, in order to keep the variance finite, see last column of table 3. In the following we consider the GST as the benchmark for our analysis since it presents a good agreement with historical simulations, see Bormetti et al. (2007). For the daily returns under study we report in figure 2 ML estimates and their $68 \%$ confidence intervals computed from the cumulative function obtained generating 1000 bootstrap copies of the original time series. Numerical details are reported in tables 1 and 3 . The solid line in figure 2 joints the estimated values of $\operatorname{VaR}(\%)$ while the dashed lines connects the boundaries of the $68 \%$ credible/bootstrap intervals.

\section{TABLE 3 ABOUT HERE}

\section{FIGURE 2 ABOUT HERE}

At $\alpha=1 \%$ the results obtained with $\boldsymbol{\sigma}^{2}$-PPM and $\boldsymbol{\sigma}^{2}$-CP are the ones in best agreement with the GST distribution, while Normal and $\boldsymbol{\mu}$-PPM underestimate VaR. The situation is different if we consider $\alpha=5 \%$. In this case $\boldsymbol{\mu}$-PPM is the only one in agreement with the GST distribution, while $\boldsymbol{\sigma}^{2}$-PPM and $\boldsymbol{\sigma}^{2}$-CP overestimate VaR.

For time horizons longer than one-day, we focus mainly on the 10-day holding period, as required by Basel Committee for the computation of regulatory VaR. Indeed, the Committee prescribes the following formula for the calcula- 
tion of regulatory capital for market risk

$$
M R C_{t}=\max \left(\frac{h}{60} \sum_{i=1}^{60} \Lambda_{t-i}^{0.01}(10), \Lambda_{t}^{0.01}(10)\right)
$$

where $M R C_{t}$ is the market risk capital at time $t, \Lambda_{t-i}^{0.01}(10)$ is VaR (not normalized by $W_{0}$, see equation (1)) at $\alpha=1 \%$ for 10 -day ahead computed using past returns up to time $t-i$ and $h$ is a penalty multiplier ranging from 3 to 4, fixed according to the traffic light rule, see Basel Committee (2006) for more details. From the original daily returns time series we compute the series of non-overlapping 10-day returns to have the 10-day VaR forecast for both $\boldsymbol{\mu}$-PPM and $\boldsymbol{\sigma}^{2}$-PPM. This approach can be easily generalised to an arbitrary holding period.

\section{TABLE 4 ABOUT HERE}

In table 4 we report the estimated 10-day ahead $\operatorname{VaR}(\%)$ for the standard significance levels $\alpha=1 \%$ and $\alpha=5 \%$ with their $68 \%$ credible intervals. Basel regulations recommend to use the so-called square-root-of-time rule to obtain the 10-day VaR from the one-day VaR. However, as already pointed out in Daníelsson et al. (1998), this rule strongly depends on the assumption of normally i.i.d. returns. The ratio between 10-day and one-day VaR estimated with parametric PPMs is readily computed and indeed our results confirm a statistically significative violation of the square root scaling law, thus highlighting the ability of our approach to better capture the properties of returns time series.

\subsection{Sensitivity Analysis and Outliers Detection}

Although the choices of parameters value used in the previous sections represent our prior knowledge and beliefs about the problem, it is illustrative to assess the sensitivity of the results to other choices of the hyperparameters.

We first consider the dependence of VaR estimates on the value of the $c$ in the cohesion function in (41). In figure 3 we plot the results for the $\boldsymbol{\mu}$-PPM model for $\alpha=1 \%$ and $c=0.1,0.5,1,5,10,50$.

\section{FIGURE 3 ABOUT HERE}

Note that for MIB30 and SRG.MI, the results are remarkably robust for a wide range of values of $c$. For MB.MI and LTO.MI the estimated value of VaR exhibits a slightly decreasing trend.

To study the sensitivity of our results to the parameters $\lambda_{0}$ and $\nu_{0}$ of the 
Inverted Gamma distribution it is convenient to re-express them in terms of a common parameter $a$. We set $\lambda_{0}=a(a+1)$ and $\nu_{0}=2+a$ in order to obtain prior expectation and variance for $\sigma^{2}$ both equal to $a$. In figure 4 we present the results for $a=0.0001,0.001,0.01,0.1,1$. For $a=1$ we have completely out-of-scale results.

\section{FIGURE 4 ABOUT HERE}

In this paper we use $a=0.01$, reflecting past knowledge regarding the problem at hand. For this reason we focus on a region around this value. The highest stability is reached when the PPM approach is applied to the vector of variances. In fact for $a \leq 0.01$ the results within the $68 \%$ credible intervals are almost identical. The $\boldsymbol{\mu}$-PPM is less stable. These results are confirmed in figure 5 where we plot the posterior distributions for LTO.MI $\alpha=1 \% \mathrm{VaR}$, with $a=0.0001,0.001,0.01,0.1$.

\section{FIGURE 5 ABOUT HERE}

We note that for $a=0.0001$ and $a=0.001$ the distributions obtained with the $\boldsymbol{\sigma}^{2}$-PPM are almost overlapping. A similar behaviour is observed for the other three time series.

We also explored separately the role played by $\lambda_{0}$ and $\nu_{0}$ and we found that $\lambda_{0}$ assumes a crucial role. We than tested the effects of an hyperprior over the scale parameter $\lambda_{0}$. We considered various combination of the $\eta$ and $\phi$ parameters, as given in equation (8). For all the tested values we were not able to achieve a reasonable sensitivity reduction. For the sake of parsimony we do not report here our results.

\section{FIGURE 6 ABOUT HERE}

In order to identify outlying points we apply the procedure described in section 4. The results are reported in figure 6. Returns corresponding to atypical values are represented by a small triangle (gains) or a small circle (losses). Their identification represents a by-product result of our approach to VaR computation. It could be interesting to investigate the economical reasons responsible for the anomalous fluctuations of assets price, along the same lines depicted in De Giuli et al. (2009). Finally it is interesting to investigate the

stability of our procedure with respect to the value of $c$. Table 5 summarizes the results of our analysis when increasing $c$ from 0.1 until 50 .

\section{TABLE 5 ABOUT HERE}

The outliers identification algorithm appears to be quite stable. As expected, on average the number of outlying points increases for increasing values of $c$. 


\subsection{Backtesting Procedures}

The current internal model verification procedure of the Basel II framework consists of recording the daily exceptions of the $1 \%$ VaR over the last year. We apply standard coverage tests to assess the accuracy of our VaR model; in particular we consider the unconditional coverage (UC) test by Kupiec (1995) and the conditional coverage (CC) one by Christoffersen (1998). Kupiec's test focuses on whether the actual number of VaR exceptions is equal to their expected number. Assuming that the probability of observing an exception is $p$, the number of exceptions out of a sample of $N$ observation follows a Binomial distribution $\operatorname{Bin}(N, p)$. The null hypothesis $p=\alpha$ can be assessed by using the following generalised likelihood ratio test

$$
L R_{U C}=-2 \log \left[(1-\alpha)^{N-n} \alpha^{n}\right]+2 \log \left[(1-n / N)^{N-n}(n / N)^{n}\right]
$$

where $n$ is the observed number of exceptions. This quantity is asymptotically distributed chi-square with one degree of freedom under the null hypothesis, and allows us to reject the model at $5 \%$ significance level when $L R_{U C}>3.84$. The $L R_{U C}$ can be extended to test the serial independence of deviations, introducing a deviation indicator which is equal to 0 if $\mathrm{VaR}$ is not exceeded and 1 otherwise. We consider the following combined test statistics (Christoffersen's test)

$$
\begin{aligned}
L R_{C C} & =L R_{U C}+L R_{I N D} \\
L R_{I N D} & =-2 \log \left[(1-n / N)^{N_{00}+N_{10}}(n / N)^{N_{01}+N_{11}}\right] \\
& +2 \log \left[\left(1-\pi_{0}\right)^{N_{00}} \pi_{0}^{N_{01}}\left(1-\pi_{1}\right)^{N_{10}} \pi_{1}^{N_{11}}\right]
\end{aligned}
$$

where $N_{i j}$ is the number of days in which state $j$ occurred in one day while was $i$ the previous day, and $\pi_{i}$ the probability of observing an exception conditional on the state $i$ the previous day, that is $\pi_{0}=N_{01} /\left(N_{00}+N_{01}\right)$ and $\pi_{1}=$ $N_{11} /\left(N_{10}+N_{11}\right)$. The null hypothesis for the independence test states that the violation occurred one day does not depend upon the indicator state the previous day. Under this hypothesis, the $L R_{C C}$ statistics is distributed chisquare with two degrees of freedom and the VaR model will be rejected at $5 \%$ significance level if $L R_{C C}>5.99$.

We perform the validation tests described above to all our data series, using a rolling window of returns to compute the VaR estimate by our models and comparing this estimate with the realized return. More precisely, at each stage $J=1, \ldots, N$, our Gibbs sampling algorithms compute the ex ante VaR estimate $\operatorname{VaR}_{M A X_{J}}^{\alpha}$ using the returns $y_{i}$ with $i=J, \ldots, M A X_{J}$; then we check $V a R_{M A X_{J}}^{\alpha}$ against the ex post realized return $y_{M A X_{J}+1}$. An exception occurs when $y_{M A X_{J}+1}<-V a R_{M A X_{J}}^{\alpha}$. A state indicator $I_{J}$ is set equal to 1 if we register an exception, and equal to 0 otherwise. This way we obtain the numbers 
$n$ and $N^{i j}$ needed to compute the $L R_{C C}$ statistics.

\section{TABLE 6 ABOUT HERE}

Choosing $M A X_{J}=J+744$, we are able to use all the information from our original series of 1000 returns and to obtain $N=255$ VaR estimates, roughly corresponding to one trading year. In table 6 are reported the results for the LTO.MI series. Our VaR models perform reasonably well with respect to both Kupiec's and Christoffersen's tests; the only exception is represented by the $\boldsymbol{\sigma}^{2}$-PPM model with $\alpha=5 \%$, which produced $n=5$ exceptions, fairly low with respect to the expected number $\mathbb{E}[n]=255 \times 0.05 \approx 13$. The reason of this pitfall has to be located in the behaviour of our returns series; actually, an empirical study of that series has shown that the associated high frequency volatility decreases almost monotonously with time. Since the algorithm is trained with those returns corresponding to the high volatility regime and is tested against returns in a low volatility regime, this consequently results in a quite conservative evaluation of VaR. A similar behaviour is noticed also for the other series.

\section{Concluding Remarks and Future Research}

In this paper we have presented a novel Bayesian methodology for VaR computation based on parametric PPMs. The main advantages of our approach are that it allows us to remain in the Normal setting, to identify anomalous observations and to obtain a closed-form expression for the VaR measure. This expression generalizes the standard parametric formula that is used in the literature under the normality assumption. By means of PPMs we induce a clustering structure over the vector of means ( $\boldsymbol{\mu}$-PPM) and we find the best agreement with ML approaches for significance level of order $5 \%$. For lower values of $\alpha$ we obtained the best result by applying the PPMs to the vector of variances $\left(\boldsymbol{\sigma}^{2}-\mathrm{PPM}\right)$.

We are currently working on the extension of the $\boldsymbol{\sigma}^{2}$-PPM approach to the portfolio analysis. The increase in the number of assets translates into an augmented dimensionality of the problem. In fact, the vector of variances is now replaced by the vector of covariance matrices. In order to reduce the number of involved parameters we are exploring several filtering techniques, see e.g. Laloux et al. (1999), Plerou et al. (1999), and Tumminello et al. (2007). 


\section{Acknowledgements}

The authors are thankful to Guido Montagna and Oreste Nicrosini for helpful comments and suggestions. Maria Elena De Giuli and Claudia Tarantola acknowledge the University of Pavia for partial support.

\section{References}

Antoniak, C.E., 1974. Mixtures of Dirichlet processes with applications to Bayesian nonparametric problems. Ann. Statist. 2, 1152 - 1174.

Barry, D., Hartigan, J.A., 1992. Product partition models for change point problems. Ann. Statist. 20, 260 - 279.

Basel Committee on Banking Supervision, 2006. Basel II: International convergence of capital measurement and capital standards. A revised framework.

Bormetti, G., Cisana. E., Montagna, G., Nicrosini, O., 2007. A non-Gaussian approach to risk measures. Physica A 376, 532 - 542.

Bush, C.A., MacEachern, S.N., 1996. A semiparametric Bayesian model for randomised block designs. Biometrika 83, 275 - 285.

Chang, G., Feigenbaum, J., 2008. Detecting log-periodicity in a regimeswitching model of stock returns. Quant. Finance 8, 723 - 738.

Christoffersen, P.F., 1998. Evaluating interval forecasts. Int. Econ. Rev. 39, 841-862.

Daníelsson, J., Hartmann, P., de Vries, C.G., 1998. The cost of conservatism: Extreme returns, Value-at-Risk, and the basle "multiplication factor". Risk January.

De Giuli, M.E., Maggi, M.A., Tarantola, C., 2009. Bayesian outlier detection in Capital Asset Pricing Model. Stat. Model., to appear.

Gelfand, A.E., Smith, F.M., 1990. Sampling-based approaches to calculating marginal densities. J. Amer. Statist. Assoc. 85, 398 - 409.

Geman, S., Geman, D., 1984. Stochastic relaxation, Gibbs distributions, and Bayesian restoration of images. IEEE Trans. Pattn Anal. Mach. Intell. 6, $721-741$.

Hartigan, J.A., 1990. Partition models. Commun. Statist. Theory Methods 19, $2745-2756$.

Hastings, W.K., 1970. Monte Carlo sampling methods using Markov chains and their applications. Biometrika 57, 97 - 109.

Jorion, P. 2001. Value at Risk: The new benchmark for managing financial risk. McGraw-Hill: New York.

Laurent, L., Cizeau, P., Bouchaud, J.P., Potters, M. 1999. Noise dressing of financial correlation matrices. Phys. Rev. Lett. 83, 1467 - 1470.

Kupiec, P.H., 1995. Techniques for verifying the accuracy of risk measurement models. J. Derivatives 3, 73-84. 
Liu, J.S., 1996. Nonparametric hierarchical Bayes via sequential inputations. Ann. Statist. 24, 911 - 930.

Loschi, R.H., Cruz, F.R.B., Iglesias, P.L., Arellano-Valle, R.B., 2003. A Gibbs sampling scheme to the product partition model: An application to changepoint problems. Comp. Oper. Res. 30, 463 - 482.

Loschi, R.H., Iglesias, P.L., Arellano-Valle, R.B., Cruz, F.R.B., 2007. Full predictivistic modeling of stock market data: Application of change point problems. Eur. J. Oper. Res. 180, 282 - 291.

Loschi, R.H., Cruz, F.R.B., Takahashi, R.H.C., Iglesias, P.L., Arellano-Valle, R.B., MacGregor Smith, J. , 2008. A note on Bayesian identification of change points in data sequences. Comp. Oper. Res. 35, $156-170$.

Manganelli, S., Engle, R.F. 2004. In Risk Measures for the 21st Century, Szegö G. Ed. Wiley: Chichester.

Mattedi, A.P., Ramos, F.M., Rosa, R.R., Mantegna, R.N. , 2004. Value-at-risk and Tsallis statistics: Risk analysis of the aerospace sector. Physica A $\mathbf{3 4 4}$, $554-561$.

Metropolis, N., Rosenbluth, A.W., Rosenbluth, M.N., Teller, A.H., Teller, E. 1953. Equations of state calculations by fast computing machines. J. Chem. Phys. 21, 1087 - 1091.

Mina, J., Xiao, J.Y., 2001. Return to RiskMetrics. The evolution of a standard. RiskMetrics Group, New York.

Plerou, V., Gopikrishnan, P., Rosenow, B., Amaral, L.A.N., Stanley, H. E. (1999). Universal and nonuniversal properties of cross correlations in financial time series. Phys. Rev. Lett. 83, 1471 - 1474.

Quintana, F.A., Iglesias, P.L., 2003. Bayesian clustering and product partition models. J. Roy. Statist. Soc. B 65, 557 - 574.

Quintana, F.A., Iglesias, P.L., Bolfarine, H., 2005. Bayesian identification of outliers and change-points in measurement error models. Adv. Complex Syst. 8, $433-449$.

Smith, B. (2001) Bayesian Output Analysis program: version 1.0.0. Dept. of Biostatistics. University of Iowa, USA. Available at http://www.publichealth.uiowa.edu/boa.

Tarantola, C., Consonni, G., Dellaportas, P., 2008. Bayesian clustering for row effects models. JSPI 138, 2223 - 2235.

Tumminello, M., Lillo, F., Mantegna, R.N. 2007. Kullback-Leibler distance as a measure of the information filtered from multivariate data. Phys. Rev. E 76, 031123. 
Table 1

Daily estimated VaR (\%) values at $5 \%$ and $1 \%$ significance level with $68 \%$ credible intervals.

\begin{tabular}{lcccccc}
\hline \hline \multirow{2}{*}{$\operatorname{VaR}(\%)$} & \multicolumn{3}{c}{$\alpha=5 \%$} & & \multicolumn{3}{c}{$\alpha=1 \%$} \\
\cline { 2 - 7 } & $\boldsymbol{\mu}$-PPM & $\boldsymbol{\sigma}^{2}-\mathrm{PPM}$ & $\boldsymbol{\sigma}^{2}-\mathrm{CP}$ & $\boldsymbol{\mu}$-PPM & $\boldsymbol{\sigma}^{2}-\mathrm{PPM}$ & $\boldsymbol{\sigma}^{2}-\mathrm{CP}$ \\
\hline MIB30.MI & $1.45_{-0.05}^{+0.05}$ & $1.74_{-0.12}^{+0.11}$ & $1.76_{-0.01}^{+0.01}$ & $2.07_{-0.06}^{+0.06}$ & $2.48_{-0.17}^{+0.16}$ & $2.49_{-0.01}^{+0.01}$ \\
LTO.MI & $2.08_{-0.07}^{+0.07}$ & $2.78_{-0.16}^{+0.15}$ & $2.66_{-0.02}^{+0.02}$ & $2.95_{-0.09}^{+0.09}$ & $3.94_{-0.21}^{+0.21}$ & $3.78_{-0.03}^{+0.03}$ \\
MB.MI & $1.91_{-0.08}^{+0.07}$ & $2.40_{-0.12}^{+0.12}$ & $2.36_{-0.01}^{+0.01}$ & $2.72_{-0.11}^{+0.11}$ & $3.40_{-0.17}^{+0.17}$ & $3.35_{-0.02}^{+0.02}$ \\
SRG.MI & $1.58_{-0.05}^{+0.05}$ & $1.97_{-0.13}^{+0.12}$ & $2.01_{-0.01}^{+0.01}$ & $2.26_{-0.06}^{+0.06}$ & $2.81_{-0.17}^{+0.17}$ & $2.87_{-0.02}^{+0.02}$ \\
\hline
\end{tabular}

Table 2

Posterior mean of the number of clusters and relative weight of the largest cluster for $\boldsymbol{\mu}$-PPM and $\boldsymbol{\sigma}^{2}$-PPM.

\begin{tabular}{lcccc}
\hline \hline & \multicolumn{2}{c}{ Number of Clusters } & \multicolumn{2}{c}{ Largest Cluster Weight } \\
\cline { 2 - 5 } & $\boldsymbol{\mu}$-PPM & $\boldsymbol{\sigma}^{\mathbf{2}}$-PPM & $\boldsymbol{\mu}$-PPM & $\boldsymbol{\sigma}^{2}$-PPM \\
MIB30.MI & 3.11 & 3.39 & 0.986 & 0.990 \\
LTO.MI & 5.02 & 4.52 & 0.963 & 0.944 \\
MB.MI & 4.11 & 3.72 & 0.968 & 0.970 \\
SRG.MI & 3.44 & 3.59 & 0.984 & 0.978 \\
\hline
\end{tabular}

Table 3

Daily ML estimated $\operatorname{VaR}(\%)$ values at $5 \%$ and $1 \%$ significance level with $68 \%$ bootstrap intervals. In the last column we report central value and $68 \%$ bootstrap interval for the tail index $\nu$ of the GST.

\begin{tabular}{lcccc|c}
\hline \hline \multirow{2}{*}{$\operatorname{VaR}(\%)$} & \multicolumn{2}{c}{$\alpha=5 \%$} & \multicolumn{3}{c}{$\alpha=1 \%$} \\
\cline { 2 - 6 } & Normal & Student- $t$ & Normal & Student- $t$ & $\nu$ \\
\hline MIB30.MI & $1.38_{-0.07}^{+0.05}$ & $1.27_{-0.06}^{+0.04}$ & $1.95_{-0.09}^{+0.07}$ & $2.22_{-0.10}^{+0.09}$ & $4.16_{-0.48}^{+0.43}$ \\
LTO.MI & $2.50_{-0.15}^{+0.14}$ & $2.15_{-0.09}^{+0.08}$ & $3.55_{-0.20}^{+0.19}$ & $4.05_{-0.22}^{+0.19}$ & $3.26_{-0.30}^{+0.28}$ \\
MB.MI & $2.07_{-0.09}^{+0.06}$ & $1.89_{-0.07}^{+0.05}$ & $2.95_{-0.11}^{+0.09}$ & $3.37_{-0.14}^{+0.12}$ & $3.93_{-0.38}^{+0.35}$ \\
SRG.MI & $1.62_{-0.08}^{+0.05}$ & $1.48_{-0.07}^{+0.04}$ & $2.32_{-0.10}^{+0.08}$ & $2.65_{-0.12}^{+0.11}$ & $3.97_{-0.44}^{+0.44}$ \\
\hline
\end{tabular}


Table 4

Estimated 10-day $\operatorname{VaR}(\%)$ values at $5 \%$ and $1 \%$ significance level with $68 \%$ credible intervals.

\begin{tabular}{lcccc}
\hline \hline \multirow{2}{*}{$\operatorname{VaR}(\%)$} & \multicolumn{2}{c}{$\alpha=5 \%$} & \multicolumn{2}{c}{$\alpha=1 \%$} \\
\cline { 2 - 5 } & $\boldsymbol{\mu}$-PPM & $\boldsymbol{\sigma}^{2}-\mathrm{PPM}$ & $\boldsymbol{\mu}$-PPM & $\boldsymbol{\sigma}^{\mathbf{2}-\mathrm{PPM}}$ \\
\hline MIB30.MI & $4.19_{-0.40}^{+0.41}$ & $4.43_{-0.46}^{+0.46}$ & $5.98_{-0.51}^{+0.51}$ & $6.33_{-0.61}^{+0.59}$ \\
LTO.MI & $6.35_{-0.70}^{+0.69}$ & $7.59_{-0.85}^{+0.83}$ & $9.08_{-0.90}^{+0.89}$ & $10.90_{-1.13}^{+1.11}$ \\
MB.MI & $6.55_{-0.65}^{+0.65}$ & $7.03_{-0.71}^{+0.70}$ & $9.41_{-0.80}^{+0.82}$ & $10.07_{-0.91}^{+0.92}$ \\
SRG.MI & $4.49_{-0.47}^{+0.47}$ & $4.83_{-0.55}^{+0.54}$ & $6.60_{-0.59}^{+0.59}$ & $7.06_{-0.72}^{+0.70}$ \\
\hline
\end{tabular}

Figure 1. VaR posterior distribution for $\alpha=1 \%$.
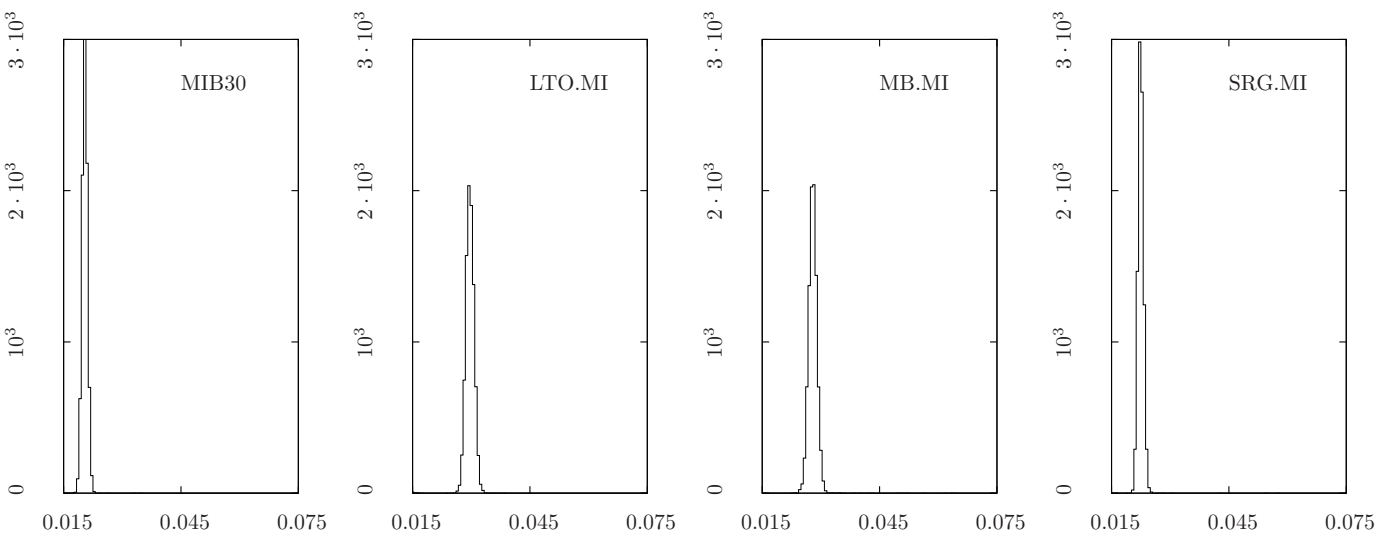

\section{$\sigma^{2}-\mathrm{PPM}$}
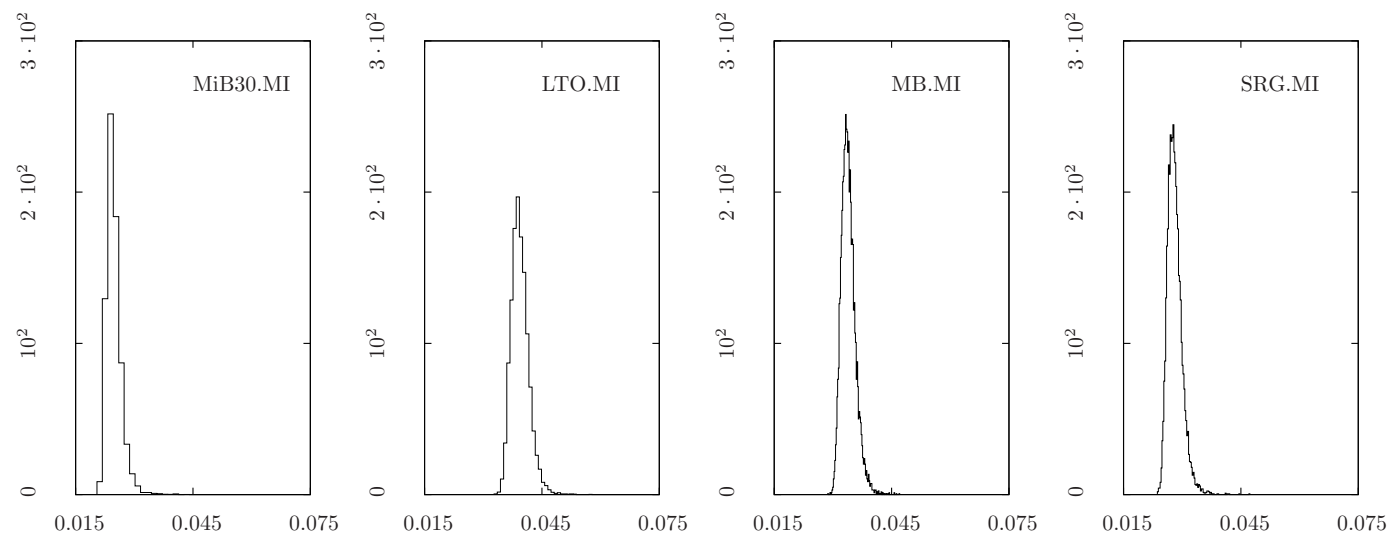
Figure 2. Comparison between classical and Bayesian estimates of $\operatorname{VaR}(\%)$. We consider two values for the levels $\alpha=1 \%$ (top panel) and $\alpha=5 \%$ (lower panel).

$$
\alpha=1 \%
$$
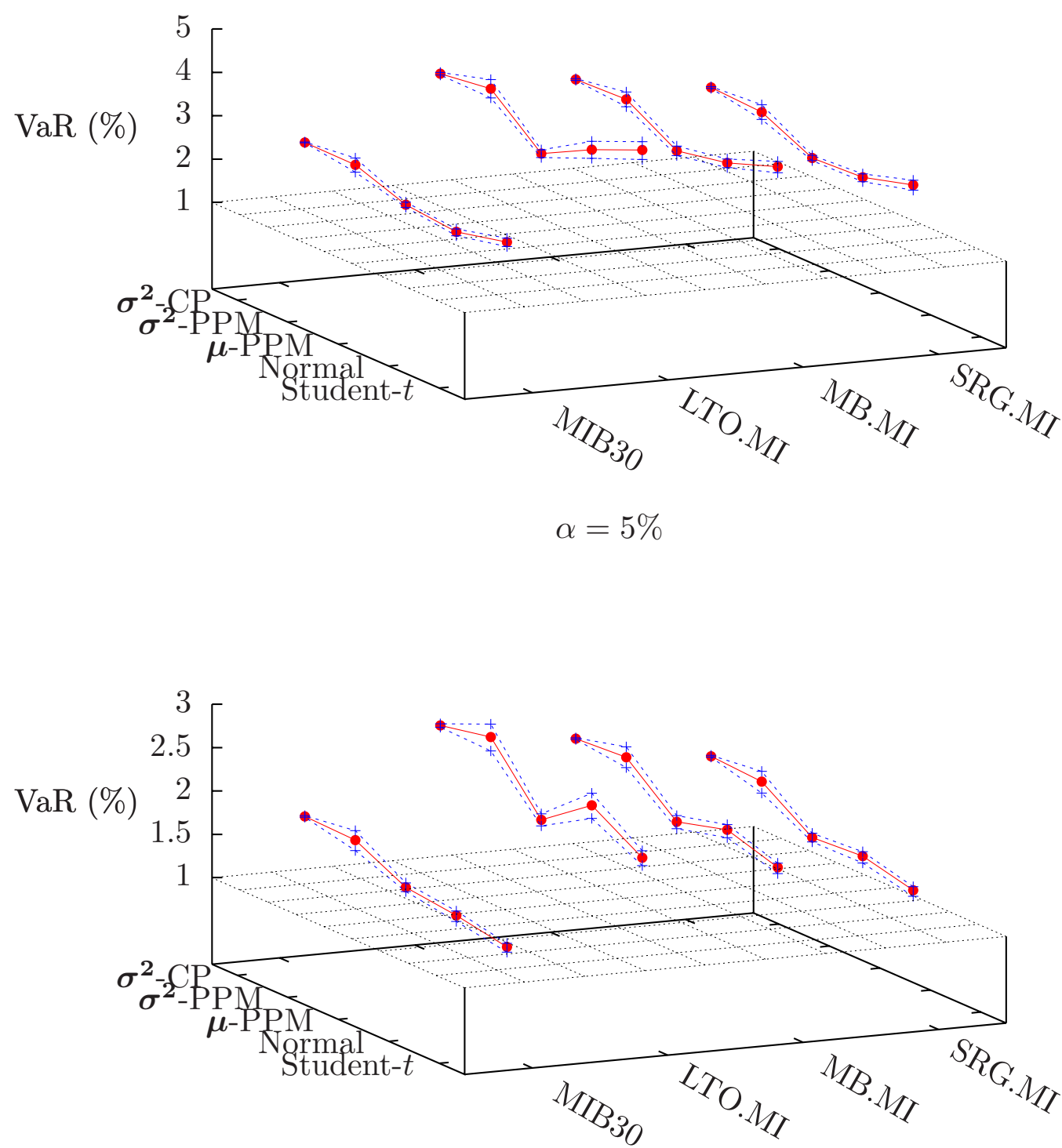
Figure 3. Sensitivity of $\alpha=1 \% \operatorname{VaR}(\%)$ estimates for the $\boldsymbol{\mu}$-PPM model with respect to the value of the hyperparameter $c$ in the cohesion function (44). The other hyperparameters assume the values quoted in the main text.

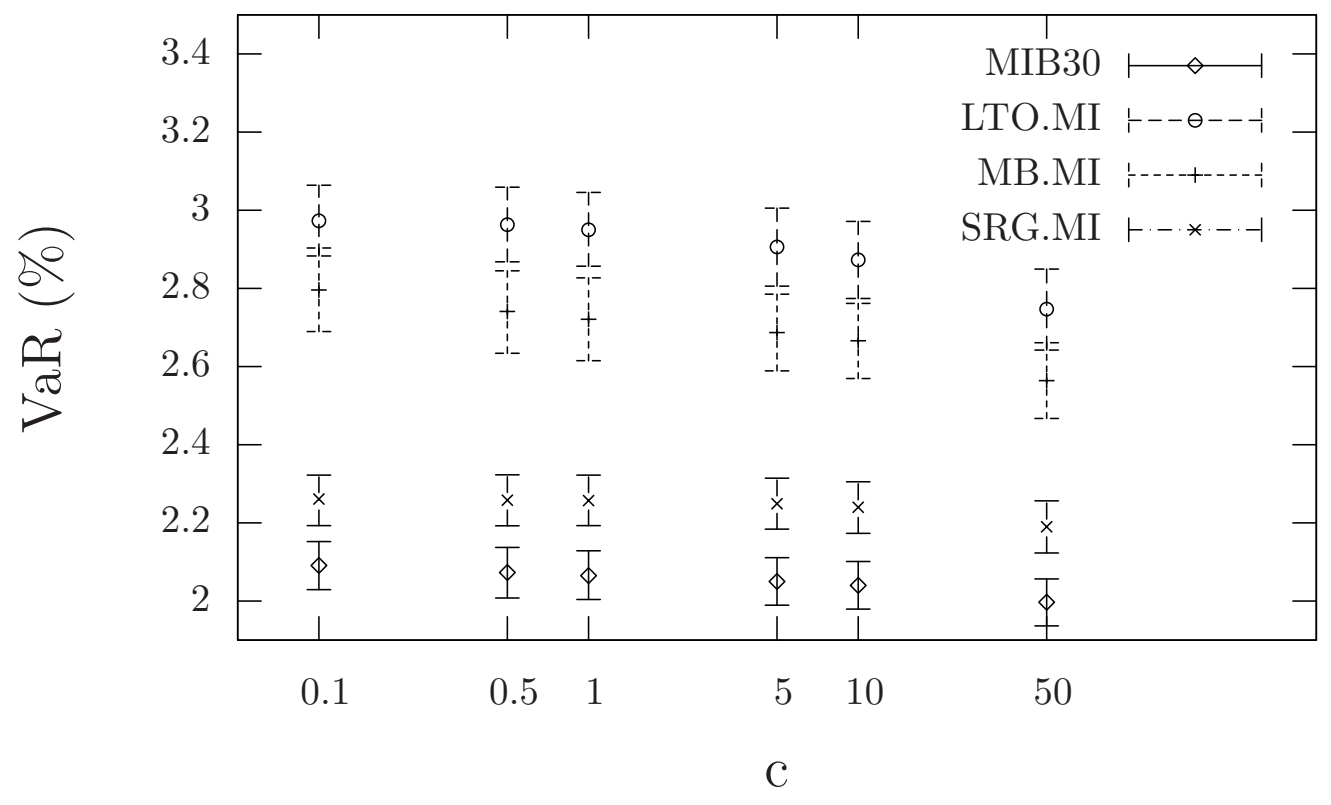


Figure 4. Sensitivity of $\alpha=1 \% \operatorname{VaR}(\%)$ estimates with respect to the value of the hyperparameters $\lambda_{0}=a(a+1)$ and $\nu_{0}=2+a$. The other hyperparameters assume the values quoted in the main text.
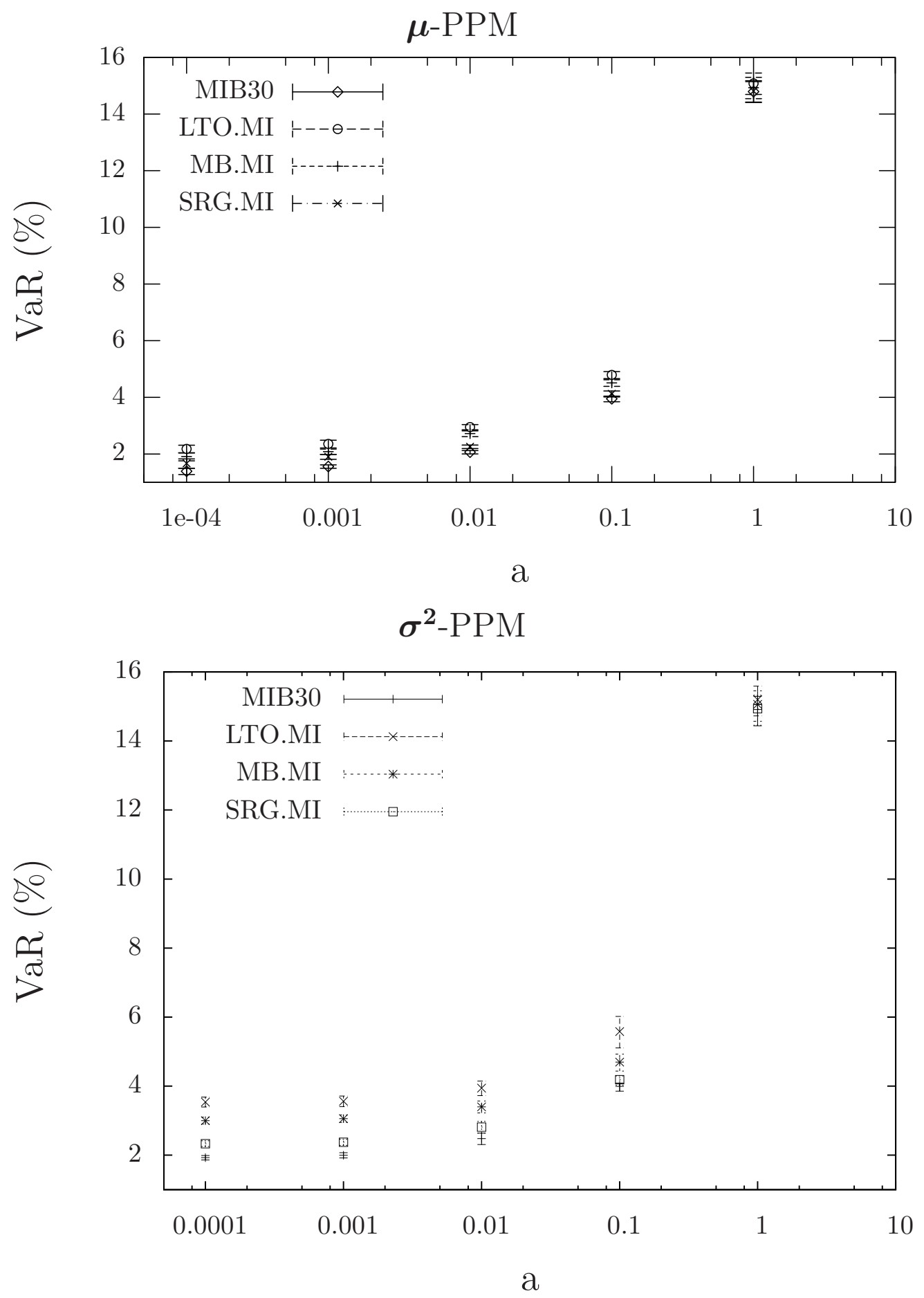
Figure 5. Posterior distributions for VaR at level $\alpha=1 \%$ for Lottomatica as a function of $a$. The other hyperparameters assume the values quoted in the main text.

$\boldsymbol{\mu}$-PPM
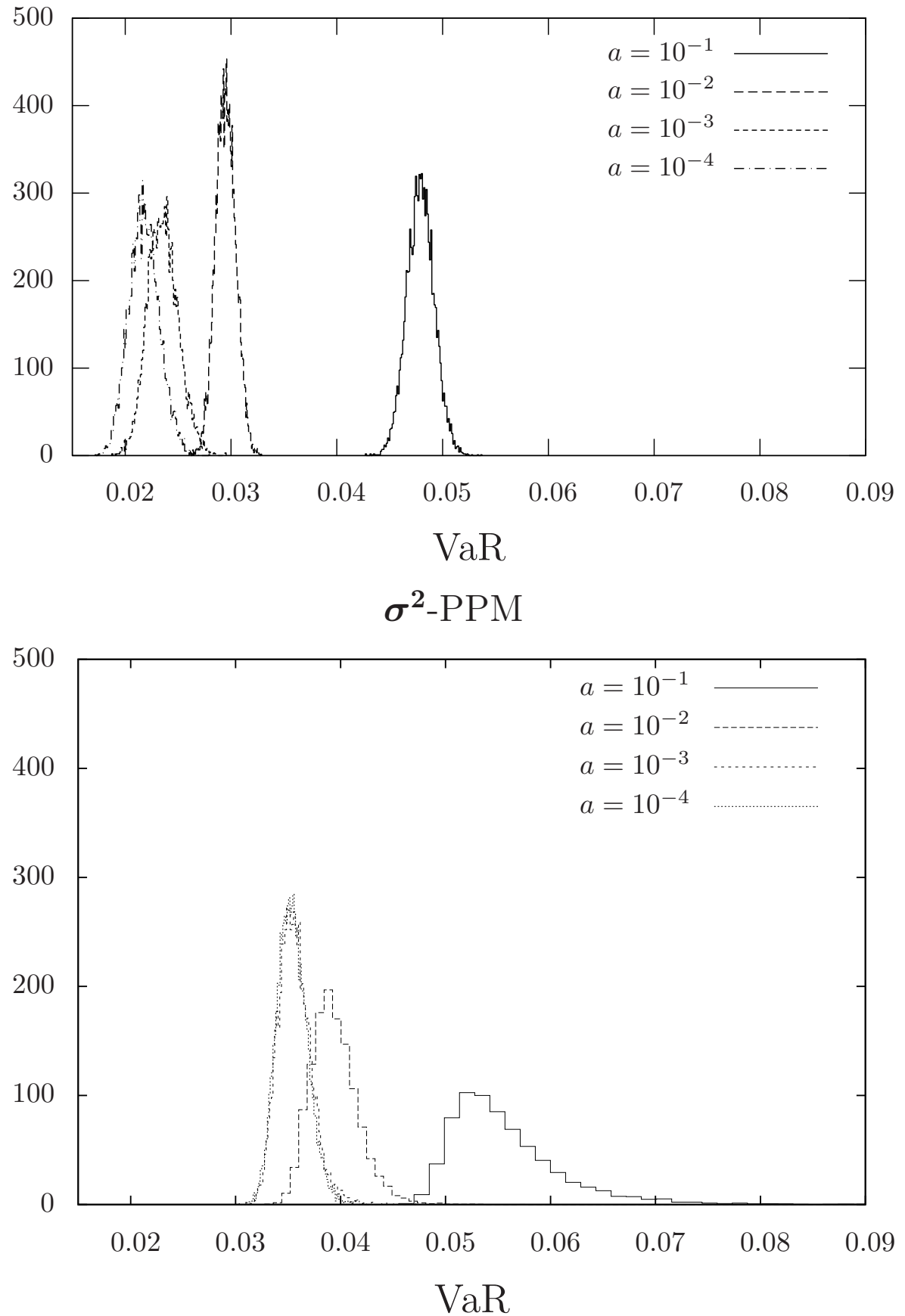
Figure 6. Detected outliers.
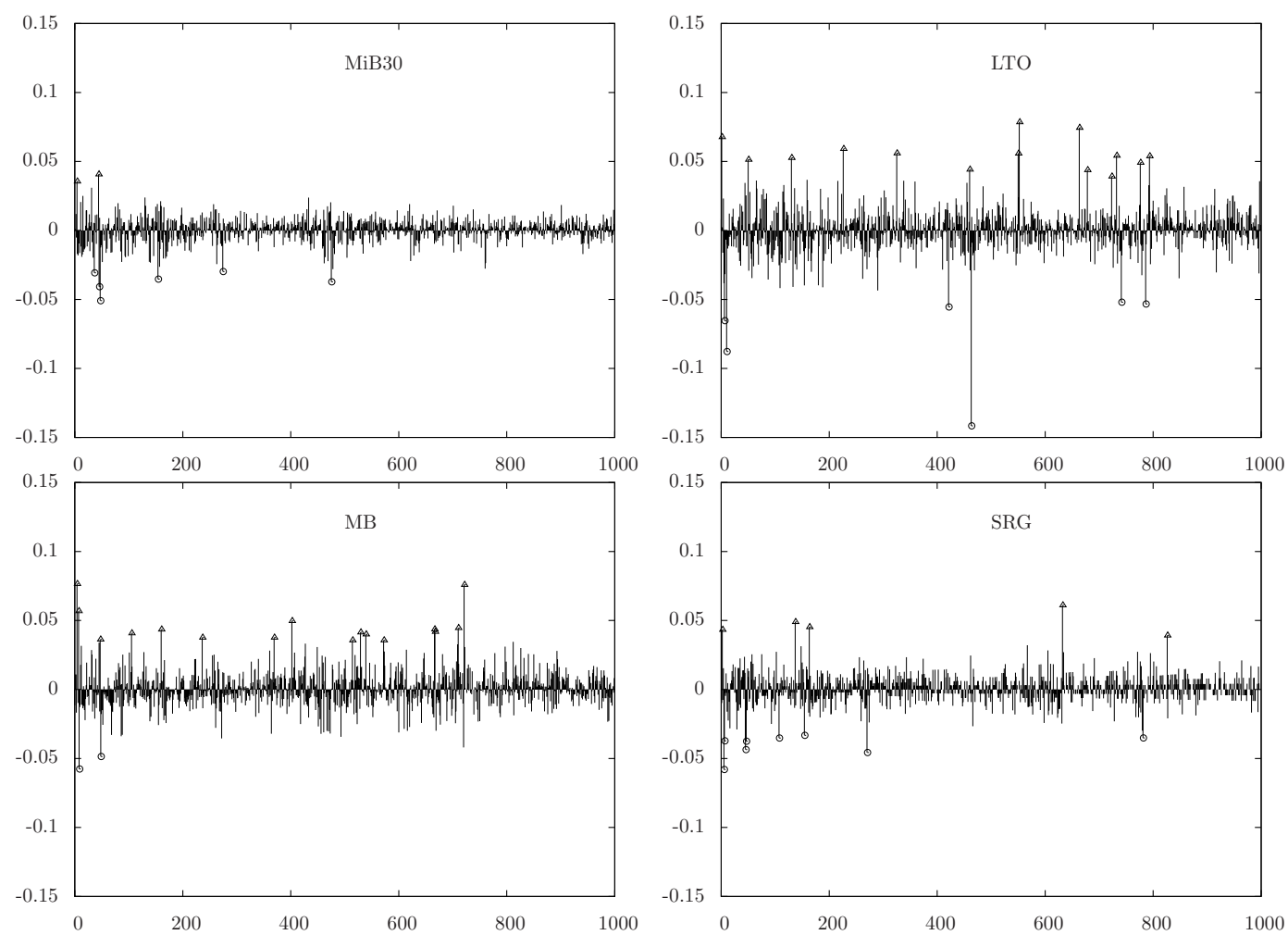
Table 5. Sensitivity analysis of outliers detection with respect to the value of $c . a, \lambda_{0}, \nu_{0}$ assume the values quoted in the main text. Subscripts ${ }_{1,2,3}$ mean the corresponding cluster being equal to $S_{1}, S_{2}$, and $S_{3}$ respectively (see section 4).

\begin{tabular}{lll}
\hline \hline $\mathrm{c}$ & \multicolumn{1}{c}{ MIB30 } & \multicolumn{1}{c}{ SRG.MI } \\
\hline 0.1 & $\left\{(46,48,155,476)_{1}(37,275)_{2}\right\}$ & $\left\{(6,7,46,47,108,155,271,782)_{1}(3,138,164,633,827)_{3}\right\}$ \\
0.5 & $\left\{(46,48,155,476)_{1}(37,275,479,761)_{2}\right\}$ & $\left\{(6,7,46,47,108,155,271,782)_{1}(3,138,164,633,827)_{3}\right\}$ \\
1 & $\left\{(37,46,48,155,275,476)_{1}(5,45)_{3}\right\}$ & $\left\{(6,7,46,47,108,155,271,782)_{1}(3,138,164,633,827)_{3}\right\}$ \\
5 & $\left\{(37,46,48,155,275,476)_{1}(5,45)_{3}\right\}$ & $\left\{(6,7,46,47,108,155,271,782)_{1}(3,138,164,633,827)_{3}\right\}$ \\
10 & $\left\{(37,46,48,155,275,476)_{1}(5,45)_{3}\right\}$ & $\left\{(6,7,46,47,108,155,271,782)_{1}(3,138,164,633,827)_{3}\right\}$ \\
50 & $\left\{(37,46,48,155,275,476,479,761)_{1}(5,32,45)_{3}\right\}$ & $\left\{(6,7,46,47,108,155,271,782)_{1}(3,138,164,633,827)_{3}\right\}$ \\
\hline $\mathrm{c}$ & & LTO.MI
\end{tabular}

$0.1\left\{(7,11,422,464,742,787)_{1}(2,51,131,227,326,461,551,553,664,679,733,777,794)_{3}\right\}$

$0.5\left\{(7,11,422,464,742,787)_{1}(2,51,131,227,326,461,551,553,664,679,733,777,794)_{3}\right\}$

$1 \quad\left\{(7,11,422,464,742,787)_{1}(2,51,131,227,326,461,551,553,664,679,724,733,777,794)_{3}\right\}$

$5 \quad\left\{(7,11,422,464,742,787)_{1}(2,51,131,227,326,461,551,553,664,679,724,733,777,794)_{3}\right\}$

$10\left\{(7,11,422,464,742,787)_{1}(2,51,131,227,326,461,551,553,664,679,724,733,777,794)_{3}\right\}$

$50\left\{(7,11,110,134,155,181,189,291,422,464,742,787)_{1}\right.$

$\left.(2,51,131,227,326,461,551,553,664,679,724,733,777,794)_{3}\right\}$

\begin{tabular}{ll}
\hline $\mathrm{c}$ & \multicolumn{1}{c}{ MB.MI } \\
\hline 0.1 & $\left\{(48,106,237,370,515,530,540,573)_{2}(5,8,161,403,667,668,711,722)_{3}\right\}$ \\
0.5 & $\left\{(9,49)_{1}(5,8,48,106,161,237,370,403,515,530,540,573,667,668,711,722)_{3}\right\}$ \\
1 & $\left\{(9,49)_{1}(5,8,48,106,161,237,370,403,515,530,540,573,667,668,711,722)_{3}\right\}$ \\
5 & $\left\{(9,49,721)_{1}(5,8,48,106,161,237,370,403,515,530,540,573,667,668,711,722,813)_{3}\right\}$ \\
10 & $\left\{(9,49,721)_{1}(5,8,48,106,161,237,370,403,515,530,540,573,667,668,711,722,813)_{3}\right\}$ \\
50 & $\left\{(9,49,721)_{1}(5,8,46,48,106,161,237,370,403,428,515,530,540,573,667,668,710,711,722,813)_{3}\right\}$ \\
\hline
\end{tabular}


Table 6

Backtesting results: the model is rejected at $5 \%$ significance level if $L R_{U C}>3.84$ (unconditional coverage test), or $L R_{C C}>5.99$ (conditional coverage test).

\begin{tabular}{lcccccc}
\hline \hline \multirow{2}{*}{ LTO.MI } & \multicolumn{3}{c}{$\alpha=1 \%$} & \multicolumn{4}{c}{$\alpha=5 \%$} \\
\cline { 2 - 7 } & \# Exceptions & $L R_{U C}$ & $L R_{C C}$ & \# Exceptions & $L R_{U C}$ & $L R_{C C}$ \\
\hline $\boldsymbol{\mu}$-PPM & 5 & 1.857 & 2.057 & 9 & 1.288 & 1.947 \\
$\boldsymbol{\sigma}^{2}$-PPM & 1 & 1.237 & 1.245 & 5 & 13.873 & 14.073 \\
\hline
\end{tabular}

